\title{
REGULAÇÃO DE GLICEMIA ATRAVÉS DE UM CONTROLADOR BI-HORMONAL POR MODOS DESLIZANTES TERMINAL NÃO-SINGULAR
}

\author{
Manuchi Martins Dansa* Victor Hugo Pereira Rodrigues** \\ Tiago Roux Oliveira* \\ * Departamento de Engenharia Eletrônica e Telecomunicações - \\ Faculdade de Engenharia, Universidade do Estado do Rio de Janeiro \\ (UERJ) - 20559-900, Rio de Janeiro, RJ, Brasil, (e-mails: \\ manuchid@gmail.com ; tiagoroux@uerj.br). \\ ** Programa de Pós-Graduação em Engenharia Elétrica - \\ COPPE-UFRJ, Universidade Federal do Rio de Janeiro - 21941-972 \\ Rio de Janeiro, RJ, Brasil (e-mail: rodrigues.vhp@gmail.com).
}

\begin{abstract}
Control algorithms for a bi-hormonal artificial pancreas are proposed in such a way that insulin and glucagon actions are incorporated, aiming to avoid hypoglicemic and hiperglicemic cases. Such control algorithms are utilized in order to ensure the blood glucose regulation in type 1 diabetic patients. The mathematical modelutilized has experimental validation and represents the glucose-insulin-glucagon dynamics. First-Order Sliding Mode Controllers with and without Boundary Layer were utilized. One desires to reach, in finite time, an equilibrium condition of the closed-loop control system, even in the presence of disturbances related to feeding. The stability proof is presented assuming that the state is known. In the simulation results, exact differentiators are used in order to recover the information associated with the unmeasured state variables of the system. At last, the performance of each control strategy is evaluated by means of numerical examples.

Resumo: Deseja-se propor algoritmos de controle para um pâncreas artificial bi-hormonal que incorpore a ação da insulina e do glucagon com a finalidade de evitar quadros de hipoglicemia e hiperglicemia. Tais algoritmos são utilizados com o intuito de garantir a regulação da glicemia em pacientes diabéticos tipo 1 . O modelo matemático utilizado tem validação experimental e representa a dinâmica glicose-insulina-glucagon. Utilizou-se o controlador por Modos Deslizantes Terminal Não Singular em suas versões contínua e descontínua. Deseja-se alcançar em tempo finito uma condição de equilíbrio do sistema de controle em malha fechada, mesmo na presença de perturbações ligadas à ingestão de alimento. A prova de estabilidade é apresentada, assumindo que o estado é conhecido. Nas simulações apresentadas, utilizaram-se diferenciadores exatos na tentativa de recuperar a informação das variáveis de estado do sistema que não são mensuradas. Por fim, são apresentados exemplos numéricos para avaliar o desempenho de cada estratégia.
\end{abstract}

Keywords: Uncertain Nonlinear Systems; Biological Mathematics; Bi-hormonal Control; Diabetes; Non-Singular Terminal Sliding Mode Control.

Palavras-chaves: Sistemas Não-Lineares Incertos; Matemática Biológica; Controle Bi-hormonal; Diabetes; Controle por Modos Deslizantes Terminal Não-Singular.

\section{INTRODUÇÃO}

O pâncreas, além de cumprir determinadas funções digestivas, secreta dois hormônios importantes: insulina e glucagon. Estes hormônios são cruciais para a regulação da concentração de glicose no sangue. Esta concentração é comumente chamada de glicemia. Quando a glicemia cresce demais, a insulina é secretada, fazendo com que a glicemia diminua e retorne a um valor que pertença à faixa de normalidade. Por outro lado, quando a glicemia diminui

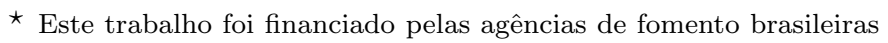
CAPES, CNPq e FAPERJ. demais, o glucagon é secretado, garantindo que a glicemia aumente e se mantenha regulada.

Em pacientes com diabetes mellitus tipo 1 (DMT1), o pâncreas não produz insulina por conta própria, o que pode trazer uma série de riscos à saúde destes pacientes Guyton and Hall (2016). Se a glicemia diminui muito, o paciente desmaia; se ela cresce demais, o paciente corre o risco de ter problemas nos olhos, nervos, e artérias. Então, muitas vezes por dia, o paciente deve furar o dedo para testar a glicemia, fazer um cálculo baseado nas refeições planejadas, se exercitar, e ajustar a injeção de insulina a fim de regular a glicemia. 
Diante deste cenário, o projeto de um dispositivo eletrônico que fosse capaz de regular a glicemia de pacientes diabéticos tipo 1 tem sido alvo de pesquisas desde meados da década de 1960 Kadish (1964). Dispositivos como este atendem pelo nome de pâncreas artificiais Cobelli et al. (2011), Bequette (2012). Um pâncreas artificial é composto por um sensor de glicose, um algoritmo e uma bomba de insulina. O sensor mede o valor correto da glicemia e envia ao algoritmo. Este, por sua vez, calcula a quantidade de hormônio que deve ser injetada no paciente através da bomba. A Figura 1 ilustra um pâncreas artificial.

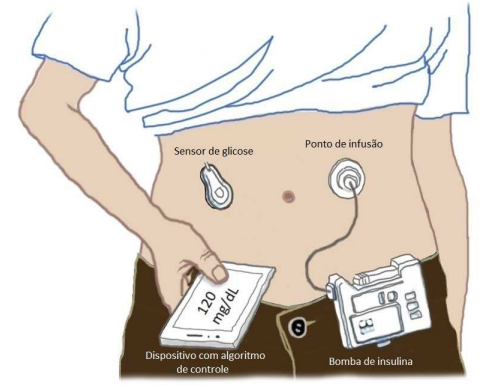

Figura 1. Pâncreas artificial mono-hormonal. Um pâncreas artificial poderia adicionar ainda uma bomba com o hormônio glucagon. Ilustração feita por Salmo Dansa.

No que diz respeito ao algoritmo, deve-se dizer que ao modelar um sistema de injeção de hormônios no corpo humano, certos requisitos como alcance em tempo finito e robustez a incertezas paramétricas, devem ser atendidos Kaveh and Shtessel (2008). Além disso, a dinâmica glicoseinsulina não é bem representada matematicamente por modelos lineares. Nesse sentido, deseja-se trabalhar com um controlador que seja capaz de alcançar o objetivo de controle desejado, em tempo finito, mesmo diante de modelagens imprecisas. O Controle por Modos Deslizantes (Sliding Mode Control - SMC) é reconhecido como uma ferramenta eficiente para projetar controladores que são robustos a incertezas paramétricas, a perturbações exógenas e a não linearidades da planta Edwards and Spurgeon (1998).

No entanto, há uma questão que tem causado divergência de opiniões entre pesquisadores deste ramo: saber se é melhor injetar apenas insulina no paciente, ou acrescentar à bomba o hormônio glucagon. Embora uma bomba monohormonal em malha fechada tenha um desempenho muito superior à terapia padrão de insulina em malha aberta, o risco de hipoglicemia ainda se mantém um desafio Bakhtiani et al. (2013).

Por outro lado, o uso de glucagon no pâncreas artificial tem se mostrado uma opção factível para prevenir e tratar a hipoglicemia El-Khatib et al. (2010), e, por isso, será utilizado neste projeto.

O principal objetivo deste artigo é propor algoritmos de controle que sejam capazes de garantir a regulação da glicemia em pacientes portadores de DMT1 através de uma bomba bi-hormonal.

\section{MODELAGEM MATEMÁTICA}

Diversos autores Castle et al. (2010), El-Khatib et al. (2010), Russell et al. (2012) acreditam que algoritmos de controle bi-hormonal garantiriam uma regulação segura da glicemia, e reduziriam significativamente o tempo gasto em estados de hipoglicemia em pacientes com DMT1. A disponibilidade de um modelo que incorpore glucagon como hormônio contra-regulatório à insulina permitiria um projeto mais eficiente de controladores bi-hormonais de glicose El-Khatib et al. (2010). Nesse sentido, um modelo mínimo estendido foi proposto Herrero et al. (2013) para incorporar o efeito do glucagon.

A fim de prezar pela simplicidade, assumiremos para o projeto dos controladores, apenas o modelo reduzido Herrero et al. (2013). Isso é possível, uma vez que algumas das dinâmicas são, em geral, mais rápidas do que a dinâmica da concentração de glicose sanguínea, da ação da insulina na produção de glicose e da ação do glucagon na produção de glicose. Desta forma, a dinâmica apresentada em Herrero et al. (2013) passa a ser representada por:

$$
\begin{aligned}
\dot{x}_{1}(t) & =-\left(S_{G}+x_{2}(t)-x_{3}(t)\right) x_{1}(t)+S_{G} G_{B}+\frac{1}{t_{\operatorname{maxG}} V} d(t), \\
\dot{x}_{2}(t) & =-p_{2}(t) x_{2}(t)+p_{2}(t) S_{I}(t)\left(u^{+}-I_{B}\right), \\
\dot{x}_{3}(t) & =-p_{3}(t) x_{3}(t)+p_{3}(t) S_{N}(t)\left(u^{-}-N_{B}\right), \\
y(t) & =x_{1}(t),
\end{aligned}
$$

onde $y(t)[\mathrm{mg} / \mathrm{dl}]$ é a variável de saída, $x_{1}(t)[\mathrm{mg} / \mathrm{dl}]$ é a concentração de glicose sanguínea, $x_{2}(t)\left[\min ^{-1}\right]$ é a ação da insulina na produção de glicose, $x_{3}(t)\left[\min ^{-1}\right]$ é a ação do glucagon na produção de glicose, $V=1.7 \mathrm{dl} / \mathrm{kg}$ é a distribuição volumétrica de glicose, $u^{+} \in \mathbb{R}^{+}[\mu \mathrm{U} / \mathrm{dl}]$ é a ação de controle dada pela concentração de insulina no plasma, $u^{-} \in \mathbb{R}^{+}[\mathrm{pg} / \mathrm{dl}]$ é a concentração de glucagon no plasma, $d(t)[\mathrm{mg} / \mathrm{kg}]$ é a concentração de glicose ingerida pelo paciente ao longo das refeiçoes diárias e $S_{G}=0,014$ $\left[\mathrm{min}^{-1}\right]$ é a efetividade da glicose por unidade de volume. A fim de prezar pela simplicidade, o parâmetro $t_{\max G}=69,6$ [min] foi feito constante, uma vez que as variações deste parâmetro em geral são muito lentas Herrero et al. (2013). As demais variáveis e parâmetros estão descritas na Tabela 1 , com as respectivas unidades e descrições. Por fim, devese ressaltar que se $u^{+} \in \mathbb{R}^{+}$e $u^{-} \in \mathbb{R}^{+}$, significa que os sinais de controle são positivos.

A descrição matemática dos parâmetros variantes no tempo é apresentada a seguir:

$$
\begin{aligned}
p_{2}(t) & =0,012 \mathbb{1}(t)-0,0081 \mathbb{1}(t-300)+0,0171 \mathbb{1}(t-720) \\
& -0,009 \mathbb{1}(t-1080), \\
p_{3}(t) & =0,017 \mathbb{1}(t)-0,001 \mathbb{1}(t-300)+0,123 \mathbb{1}(t-720) \\
& -0,122 \mathbb{1}(t-1080), \\
S_{I}(t) & =[7,73 \mathbb{1}(t)+0,82 \mathbb{1}(t-300)-1,73 \mathbb{1}(t-720) \\
& +0,91 \mathbb{1}(t-1080)] \times 10^{-4}, \\
S_{N}(t) & =[1,38 \mathbb{1}(t)+0,58 \mathbb{1}(t-300)-1,15 \mathbb{1}(t-720) \\
& +0,57 \mathbb{1}(t-1080)] \times 10^{-4}, \\
I_{B}(t) & =11,01 \mathbb{1}(t)+8,75 \mathbb{1}(t-300)-9,73 \mathbb{1}(t-720) \\
& +0,98 \mathbb{1}(t-1080), \\
N_{B}(t) & =46,30 \mathbb{1}(t)+1,83 \mathbb{1}(t-300)+11,10 \mathbb{1}(t-720) \\
& -12,93 \mathbb{1}(t-1080),
\end{aligned}
$$

onde $\mathbb{1}(t)$ representa a função degrau unitário. O deslocamento na função degrau unitário é medido em minutos. Portanto, as funções $\mathbb{1}(t-300), \mathbb{1}(t-720)$ e $\mathbb{1}(t-1080)$ indicam que os valores dos parâmetros (5)-(10) sofrem alterações às 5:00, às 12:00 e às 18:00, respectivamente. Os valores mínimos e máximos dos parâmetros (5)-(10) são dados por: 


$$
\begin{aligned}
0,0039 & \leq p_{2}(t) \leq 0,021 \\
0,016 & \leq p_{3}(t) \leq 0,139 \\
6,82 \times 10^{-4} & \leq S_{I}(t) \leq 8,55 \times 10^{-4} \\
0,81 \times 10^{-4} & \leq S_{N}(t) \leq 1,96 \times 10^{-4} \\
10,03 & \leq I_{B}(t) \leq 19,76 \\
46,30 & \leq N_{B}(t) \leq 59,23 .
\end{aligned}
$$

Durante o desenvolvimento da análise de estabilidade, os parâmetros do sistema serão considerados incertos tal que, seus limitantes são conhecidos, e descritos por

$$
\begin{aligned}
\underline{p}_{2} & <p_{2}(t)<\bar{p}_{2}, \quad \underline{p}_{3}<p_{3}(t)<\bar{p}_{3}, \\
\underline{S}_{I} & <S_{I}(t)<\bar{S}_{I}, \quad \underline{S}_{N}<S_{N}(t)<\bar{S}_{N}, \\
\underline{I}_{B} & <I_{B}(t)<\bar{I}_{B}, \quad \underline{N}_{B}<N_{B}(t)<\bar{N}_{B}, \\
\underline{t_{\operatorname{maxG}}} & <t_{\operatorname{maxG}}, \quad G_{B}<\bar{G}_{B}, \quad \underline{V}<V, \\
\underline{S_{G}} & <S_{G}<\overline{S_{G}} .
\end{aligned}
$$

Além disso, assume-se que

$$
|d(t)|<\bar{d}, \quad|\dot{d}(t)|<\dot{\bar{d}},
$$

onde $\bar{d}$ e $\dot{\bar{d}}$ são constantes positivas conhecidas para as quais (22) é satisfeita, a menos de um conjunto de medida nula no sentido de Lebesgue.

A seguir, apresentamos o objetivo de controle e a metodologia utilizada para alcançá-lo. As ferramentas exploradas com este intuito serão devidamente discutidas.

\section{OBJETIVOS DE CONTROLE E METODOLOGIA}

\subsection{Objetivo de Controle}

O objetivo deste projeto é garantir a regulação da glicemia em um paciente com DMT1. Matematicamente, isto pode ser representado a partir da estabilização do erro de saída:

$$
e(t)=G_{B}-x_{1}(t)
$$

onde $G_{B}=90 \mathrm{mg} / \mathrm{dl}$ representa o valor de referência desejado. Este valor foi escolhido de modo que a glicemia do paciente com DMT1 se mantivesse dentro dos limites considerados seguros pela comunidade médica, 80 e 100 mg/dl Guyton and Hall (2016).

\subsection{Metodologia}

Dentre todas as estratégias de controle não-linear disponíveis, escolheu-se trabalhar com controladores por modos deslizantes (Sliding Modes Control - SMC). A principal vantagem do SMC é a não necessidade de se conhecer precisamente o modelo matemático do sistema a ser controlado. Para o projeto do controlador, basta que os limitantes superiores e inferiores dos parâmetros da planta sejam conhecidos. O conhecimento dos majorantes para as perturbações também é exigido. Nesse sentido, pode-se dizer que não há problema em se trabalhar com uma planta que esteja sujeita a incertezas paramétricas e perturbações exógenas.

\section{O ATUADOR BI-HORMONAL E ALIMENTAÇÃO}

\subsection{Atuador Bi-hormonal}

Controladores por modos deslizantes têm sempre duas ações de controle: uma ação de controle positiva, $u^{+}$, e uma ação de controle negativa, $u^{-}$. Neste projeto, a ação de controle positiva é representada pela quantidade de insulina injetada no sangue, enquanto a ação de controle negativa é representada pela quantidade de glucagon injetada no sangue. O algoritmo decide qual hormônio deve ser injetado no paciente a cada momento, e esta decisão satisfaz a seguinte regra:

$$
\begin{aligned}
& u^{+}=\left\{\begin{array}{ll}
\varrho, & \text { se } u>0 \quad(\operatorname{sgn}(\sigma)<0) \\
0, & \text { caso contrário }
\end{array},\right. \\
& u^{-}=\left\{\begin{array}{ll}
\varrho, & \text { se } u<0 \quad(\operatorname{sgn}(\sigma)>0) \\
0, & \text { caso contrário }
\end{array},\right.
\end{aligned}
$$

onde $\varrho$ representa a função de modulação do controlador, e $\sigma$ representa a variável de deslizamento. Observando-se as regras (24)-(25), percebe-se que insulina e glucagon nunca são administrados simultaneamente.

\subsection{Perturbação - Alimentação}

Neste projeto, deseja-se que a glicemia do paciente seja regulada ao longo de um período de 24 horas, o que equivale a 1.440 minutos. Neste contexto, é necessário que o paciente mantenha uma dieta regular que consiste em três refeições diárias, previstas para os seguintes horários: 5:00, 12:00 e 18:00. A modelagem da ingestão de alimentos foi proposta em Kaveh and Shtessel (2008), como:

$$
\begin{aligned}
d(t)= & 80 e^{-0,5(t-300)} \mathbb{1}(t-300)+100 e^{-0,5(t-720)} \mathbb{1}(t-720) \\
& +70 e^{-0,5(t-1080)} \mathbb{1}(t-1080),
\end{aligned}
$$

onde $d(t)$ pode ser encarado como uma perturbação exógena do sistema. Desse modo, assume-se que cada refeição pode representar uma taxa diferente de aparecimento de glicose no sangue. A Figura 2 ilustra o efeito que a alimentação do paciente tem sobre sua glicemia.

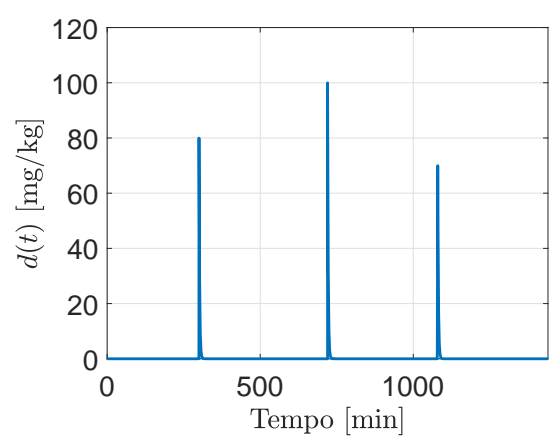

Figura 2. Concentração de glicose ingerida pelo paciente ao longo das refeições diárias.

Assim sendo, observemos o sistema (1)-(3). Nota-se que a perturbação $d(t)$ aparece na equação (1), enquanto as ações positiva, $u^{+}$, e negativa, $u^{-}$, de controle aparecem nas equações (2) e (3). Então, diz-se que as perturbações são descasadas Edwards and Spurgeon (1998). Isto gera uma dificuldade adicional no projeto do controlador, uma vez que o ganho de controle terá que majorar a perturbação e suas derivadas em amplitude.

\section{O DIFERENCIADOR EXATO}

Diferenciadores exatos são baseados em controladores por modos deslizantes de alta ordem. Este tipo de ferramenta é 
Tabela 1. Descrição dos parâmetros do modelo mínimo estendido.

\begin{tabular}{|l|l|}
\hline Parâmetros & Descrição \\
\hline \hline$S_{G}\left[\mathrm{~min}^{-1}\right]$ & efetividade da glicose por unidade de distribuição de volume \\
\hline$G_{B}[\mathrm{mg} / \mathrm{dl}]$ & concentração basal de glicose \\
\hline$t_{\max G(t)[\mathrm{min}]}$ & tempo para máxima absorção de glicose \\
\hline$V[\mathrm{dl} / \mathrm{kg}]$ & distribuição volumétrica de glicose \\
\hline$p_{2}(t)\left[\mathrm{min}^{-1}\right]$ & taxa de eliminação da insulina ativa \\
\hline$S_{I}(t)\left[\mathrm{min}^{-1}\right.$ por $\left.\mu \mathrm{U} / \mathrm{ml}\right]$ & sensibilidade de insulina \\
\hline$I_{B}(t)[\mathrm{mU} / \mathrm{dl}]$ & concentração basal de insulina \\
\hline$p_{3}(t)[\mathrm{min}-1]$ & taxa que descreve a dinâmica da ação do glucagon \\
\hline$S_{N}(t)\left[\mathrm{min}^{-1}\right.$ por $\left.\mathrm{pg} / \mathrm{ml}\right]$ & sensibilidade ao glucagon \\
\hline$N_{B}(t)[\mathrm{pg} / \mathrm{ml}]$ & concentração basal de glucagon \\
\hline
\end{tabular}

capaz de fornecer a derivada exata do erro de saída $e \in \mathbb{R}$ e garantir a atenuação de pequenos ruídos de alta frequência Levant (2003). Sua estrutura é dada por:

$$
\begin{aligned}
& \dot{\zeta}_{0}=v_{0}=-\lambda_{0} C^{\frac{1}{p+1}}\left|\zeta_{0}-e(t)\right|^{\frac{p}{p+1}} \operatorname{sgn}\left(\zeta_{0}-e(t)\right)+\zeta_{1}, \\
& \quad \vdots \\
& \dot{\zeta}_{i}=v_{i}=-\lambda_{i} C^{\frac{1}{p-i+1}}\left|\zeta_{i}-v_{i-1}\right|^{\frac{p-i}{p-i+1}} \operatorname{sgn}\left(\zeta_{i}-v_{i-1}\right)+\zeta_{i+1}, \\
& \quad \vdots \\
& \dot{\zeta}_{p}=-\lambda_{p} C \operatorname{sgn}\left(\zeta_{p}-v_{p-1}\right),
\end{aligned}
$$

onde $\lambda_{i}$ são constantes apropriadas, escolhidas de maneira recursiva; $C$ é uma constante apropriada, tal que $C \geq$ $\left|e^{(\rho)}(t)\right| ;$ o estado é dado por $\zeta=\left[\zeta_{0}, \ldots, \zeta_{\rho-1}\right]^{T} ; \mathrm{e}$ $p=\rho-1$ representa a ordem do diferenciador. Portanto, as seguintes igualdades

$$
\zeta_{0}=e(t), \quad \zeta_{i}=e^{(i)}(t), \quad i=1, \ldots, p,
$$

são estabelecidas em tempo finito Levant (2003), com a necessidade de que o sinal, $e^{(\rho)}(t)$, seja uniformemente limitado, como assumido no diferenciador por modos deslizantes de ordem superior (Higher Order Sliding Modes HOSM). Como $\rho=2$, sabendo que a planta tem dinâmica dada por (1)-(4), a partir do erro de regulação (23), é possível mostrar que a seguinte desigualdade é satisfeita:

$$
\begin{aligned}
& |\ddot{e}(t)|=\mid\left(-S_{G}^{2}-p_{2} S_{I} I_{B}+p_{3} S_{N} N_{B}\right) x_{1}(t) \\
& +S_{G} G_{B}\left(x_{2}(t)-x_{3}(t)\right)-\left(2 S_{G}+p_{2}\right) x_{1}(t) x_{2}(t) \\
& +\left(2 S_{G}+p_{3}\right) x_{1}(t) x_{3}(t)-x_{1}(t) x_{2}^{2}(t)-x_{1}(t) x_{3}^{2}(t) \\
& +2 x_{1}(t) x_{2}(t) x_{3}(t)+\frac{d(t)}{t_{\operatorname{maxG}} V}\left(S_{G}+x_{2}(t)-x_{3}(t)\right) \\
& -\frac{\dot{d}(t)}{t_{\operatorname{maxG}} V}+S_{G}^{2} G_{B}+p_{2} S_{I} x_{1}(t) u^{+}-p_{3} S_{N} x_{1}(t) u^{-} \mid \\
& \leq\left(\bar{S}_{G}^{2}+\bar{p}_{2} \bar{S}_{I} \bar{I}_{B}+\bar{p}_{3} \bar{S}_{N} \bar{N}_{B}+2 \bar{S}_{G} \bar{G}_{B}+\frac{2 \bar{d}}{\underline{t_{\operatorname{maxG}}} \underline{\mathrm{V}}}\right. \\
& \left.+\max \left\{\bar{p}_{2}, \bar{p}_{3}\right\}+\max \left\{\bar{S}_{I}, \bar{S}_{N}\right\} \varrho\right) \chi \\
& +\left(4 \bar{S}_{G}+\bar{p}_{2}+\bar{p}_{3}\right) \chi^{2}+4 \chi^{3}+\frac{\bar{S}_{G} \bar{d}+\bar{d}}{\underline{t_{\operatorname{maxG}}} \underline{\mathrm{V}}}+\bar{S}_{G}^{2} \bar{G}_{B},
\end{aligned}
$$

onde os parâmetros são majorados por (17)-(20), a perturbação, $d(t)$, e sua derivada, $\dot{e}(t)$, satisfazem $(22)$ e a norma do estado, por se tratar de um sistema biológico, obviamente é majorada, ao menos localmente, por uma constante tal que, por definição, $|x(t)|<\chi$. Assumindo que estes majorantes são constantes, e estão disponíveis para o projeto, um limitante superior para o módulo da segunda derivada do sinal de erro, $\ddot{e}(t)$, pode ser encontrado por

$$
\begin{aligned}
C & =\left(\bar{S}_{G}^{2}+\bar{p}_{2} \bar{S}_{I} \bar{I}_{B}+\bar{p}_{3} \bar{S}_{N} \bar{N}_{B}+2 \bar{S}_{G} \bar{G}_{B}+\frac{2 \bar{d}}{\frac{t_{\operatorname{maxG}} \underline{\mathrm{V}}}{\bar{p}_{2}}}\right. \\
& \left.+\max \left\{\bar{p}_{2}, \bar{p}_{3}\right\} \max \left\{\bar{S}_{I}, \bar{S}_{N}\right\} \varrho\right) \chi+\left(4 \bar{S}_{G}+\bar{p}_{3}\right) \chi^{2} \\
& +4 \chi^{3}+\frac{\bar{S}_{G} \bar{d}+\bar{d}}{\underline{t_{\operatorname{maxG}} \underline{\mathrm{V}}}}+\bar{S}_{G}^{2} \bar{G}_{B} .
\end{aligned}
$$

Ao longo deste trabalho, o seguinte diferenciador exato será utilizado:

$$
\begin{gathered}
\dot{\zeta}_{0}=v_{0}=-\lambda_{0} C^{\frac{1}{2}}\left|\zeta_{0}-e(t)\right|^{\frac{1}{2}} \operatorname{sgn}\left(\zeta_{0}-e(t)\right)+\zeta_{1}, \\
\dot{\zeta}_{1}=-\lambda_{1} C \operatorname{sgn}\left(\zeta_{1}-v_{0}\right), \\
\text { com } \lambda_{0}=5 \text { e } \lambda_{1}=3 \text { e ganho } C \text { dado em }(30) .
\end{gathered}
$$

Para a realização das simulações, os seguintes parâmetros do sistema de controle foram escolhidos: $\mathrm{p}_{2}=0,003$, $\bar{p}_{2}=0,03 ; \mathrm{p}_{3}=0,01 ; \bar{p}_{3}=0,14 ; \underline{\mathrm{S}}_{N}=0,8 \times 10^{-4}$; $\bar{S}_{N}=2 \times 10^{-4} ; \underline{S}_{I}=6,8 \times 10^{-4} ; \bar{S}_{I}=8,6 \times 10^{-4} ;$ $\bar{S}_{G}=0,015 ; \bar{N}_{B}=60 ; \bar{I}_{B}=20 ; \underline{\mathrm{V}}=1,5 ; \underline{t_{\mathrm{maxG}}}=65 ;$ $\bar{d}=110$ e $\overline{\dot{d}}=60$ e $\varrho=100$. Estes valores foram escolhidos a partir valores numéricos dos parâmetros (5)-(10) que, por sua vez, podem ser consultados em Herrero et al. (2013).

\section{CONTROLE POR MODOS DESLIZANTES TERMINAL NÃO SINGULAR: VERSÃO DESCONTÍNUA}

O controlador não-singular terminal SMC difere do SMC de primeira ordem padrão basicamente por duas razões. Em primeiro lugar, sua superfície de deslizamento é uma função não-linear das variáveis de estado do erro. Em segundo lugar, enquanto a convergência exponencial é garantida para o SMC de primeira ordem quando o sistema em malha fechada se mantém na superfície de deslizamento, o algoritmo do non-singular terminal é capaz de levar o estado para a origem em tempo finito $(e \equiv \dot{e} \equiv 0)$. Apresenta-se uma versão por realimentação de saída para o controlador apresentado em Feng et al. (2002).

Teorema 1. Considere o sistema descrito por (1)-(4) e os limitantes (17)-(20). Então, é possível que se encontre uma lei de controle por modos deslizantes $u(t)$, dada por:

$$
\begin{aligned}
u & =-\varrho \operatorname{sgn}(\sigma), \quad \varrho>0, \\
\sigma(t) & =e(t)+\frac{1}{\beta} \dot{e}^{p / q}(t),
\end{aligned}
$$

onde $\beta=2, p=5$ e $q=3$. A função de modulação $\varrho$ é uma constante suficientemente grande e a variável de deslizamento $\sigma(t)$ deve ser tal que o modo deslizante ideal em $\sigma(t)=0$ e $\dot{\sigma}(t)=0$ ocorra em tempo finito.

Prova: Considere a candidata à função de Lyapunov

$$
V(t)=\sigma^{2}(t),
$$

com $\sigma(t)$ dado em (34) e derivada temporal 


$$
\begin{aligned}
& \dot{\sigma}(t)=S_{G} x_{1}(t)+x_{1}(t) x_{2}(t)-x_{1}(t) x_{3}(t)-S_{G} G_{B}-\frac{1}{t_{\operatorname{maxG}} V} d(t)+ \\
& \frac{1}{\beta} \frac{p}{q}\left(S_{G} x_{1}(t)+x_{1}(t) x_{2}(t)-x_{1}(t) x_{3}(t)-S_{G} G_{B}-\frac{1}{t_{\operatorname{maxG}} V} d(t)\right)^{\frac{p-q}{q}} \\
& \times\left[\left(-S_{G}^{2}-p_{2} S_{I} I_{B}+p_{3} S_{N} N_{B}\right) x_{1}(t)+S_{G} G_{B}\left(x_{2}(t)-x_{3}(t)\right)\right. \\
& -\left(2 S_{G}+p_{2}\right) x_{1}(t) x_{2}(t)+\left(2 S_{G}+p_{3}\right) x_{1}(t) x_{3}(t)-x_{1}(t) x_{2}^{2}(t) \\
& -x_{1}(t) x_{3}^{2}(t)+2 x_{1}(t) x_{2}(t) x_{3}(t)+\frac{1}{t_{\operatorname{maxG}} V}\left(S_{G}+x_{2}(t)-x_{3}(t)\right) d(t) \\
& \left.-\frac{1}{t_{\operatorname{maxG}} V} \dot{d}(t)+p_{2} S_{I} x_{1}(t) u^{+}-p_{3} S_{N} x_{1}(t) u^{-}\right] .
\end{aligned}
$$

Portanto, a derivada de (35) é

$$
\begin{aligned}
& \dot{V}(t)=2\left\{\left[S_{G} x_{1}(t)+x_{1}(t) x_{2}(t)-x_{1}(t) x_{3}(t)-S_{G} G_{B}-\frac{1}{t_{\operatorname{maxG}} V} d(t)\right.\right. \\
& +\frac{1}{\beta} \frac{p}{q}\left(S_{G} x_{1}(t)+x_{1}(t) x_{2}(t)-x_{1}(t) x_{3}(t)-S_{G} G_{B}-\frac{1}{t_{\operatorname{maxG}} V} d(t)\right)^{\frac{p-q}{q}} \times \\
& \times\left(\left(-S_{G}^{2}-p_{2} S_{I} I_{B}+p_{3} S_{N} N_{B}\right) x_{1}(t)+S_{G} G_{B}\left(x_{2}(t)-x_{3}(t)\right)-x_{1}(t) x_{3}^{2}(t)\right. \\
& \left.-\left(2 S_{G}+p_{2}\right) x_{1}(t) x_{2}(t)+\left(2 S_{G}+p_{3}\right) x_{1}(t) x_{3}(t)-x_{1}(t) x_{2}^{2}(t)-x_{3}(t)\right) d(t) \\
& \left.+2 x_{1}(t) x_{2}(t) x_{3}(t)+\frac{1}{t_{\operatorname{maxG}} V}\left(S_{G}+x_{2}(t)-\frac{1}{t_{\operatorname{maxG}} V} \dot{d}(t)\right)\right] \sigma(t)+ \\
& \frac{1}{\beta} \frac{p}{q}\left(S_{G} x_{1}(t)+x_{1}(t) x_{2}(t)-x_{1}(t) x_{3}(t)-S_{G} G_{B}-\frac{1}{t_{\operatorname{maxG}} V} d(t)\right)^{\frac{p-q}{q}} \times \\
& \left.\times\left(+p_{2} S_{I} x_{1}(t) u^{+}-p_{3} S_{N} x_{1}(t) u^{-}\right) \sigma(t)\right\},
\end{aligned}
$$

que pode ser majorada por

$\dot{V}(t) \leq 2\left\{\left[S_{G}\left|x_{1}(t)\right|+\left|x_{1}(t)\right|\left|x_{2}(t)\right|+\left|x_{1}(t)\right|\left|x_{3}(t)\right|+S_{G} G_{B}\right.\right.$ $+\frac{1}{t_{\operatorname{maxG}} V}|d(t)|+\frac{1}{\beta} \frac{p}{q}\left(S_{G}\left|x_{1}(t)\right|+\left|x_{1}(t)\right|\left|x_{2}(t)\right|+\left|x_{1}(t)\right|\left|x_{3}(t)\right|\right.$

$\left.+S_{G} G_{B}+\frac{1}{t_{\operatorname{maxG}} V}|d(t)|\right)^{\frac{p-q}{q}}\left(\left(S_{G}^{2}+p_{2} S_{I} I_{B}+p_{3} S_{N} N_{B}\right)\left|x_{1}(t)\right|\right.$

$+S_{G} G_{B}\left(\left|x_{2}(t)\right|+\left|x_{3}(t)\right|\right)+\left(2 S_{G}+p_{2}\right)\left|x_{1}(t)\right|\left|x_{2}(t)\right|$

$+\left(2 S_{G}+p_{3}\right)\left|x_{1}(t)\right|\left|x_{3}(t)\right|+\left|x_{1}(t)\right|\left(\left|x_{2}(t)\right|^{2}+\left|x_{3}(t)\right|^{2}\right)$

$+2\left|x_{1}(t)\right|\left|x_{2}(t)\right|\left|x_{3}(t)\right|+\frac{1}{t_{\operatorname{maxG}} V}\left(S_{G}+\left|x_{2}(t)\right|\right.$

$\left.\left.\left.+\left|x_{3}(t)\right|\right)|d(t)|+\frac{1}{t_{\operatorname{maxG}} V}|\dot{d}(t)|\right)\right]|\sigma(t)|$

$+\frac{1}{\beta} \frac{p}{q}\left(S_{G} x_{1}(t)+x_{1}(t) x_{2}(t)\right.$

$\left.-x_{1}(t) x_{3}(t)-S_{G} G_{B}-\frac{1}{t_{\operatorname{maxG}} V} d(t)\right)^{\frac{p-q}{q}} \times$

$\left.\left(+p_{2} S_{I} x_{1}(t) u^{+} \sigma(t)-p_{3} S_{N} x_{1}(t) u^{-} \sigma(t)\right)\right\}$.

O paciente receberá insulina $\left(u^{+}=\varrho\right)$ se $u>0$, neste caso, $\operatorname{sgn}(\sigma)<0 \rightarrow \sigma=-|\sigma|$ e $u^{-}=0$. Receberá glucagon $\left(u^{-}=\varrho\right)$ se $u<0$, neste caso, $\operatorname{sgn}(\sigma)>0 \rightarrow \sigma=|\sigma|$ e $u^{+}=0$. Então, assumindo, por simplicidade, que: $(i) x_{1}(t)>1$; $(i i)$ que é sempre possível encontrar $k_{G}$ suficientemente pequeno; e $($ iii) $\left(S_{G} x_{1}(t)+x_{1}(t) x_{2}(t)-x_{1}(t) x_{3}(t)-S_{G} G_{B}+\right.$ $\left.-\frac{1}{t_{\mathrm{maxG}} V} d(t)\right)^{\frac{p-q}{q}} \geq k_{G}\left(S_{G} G_{B}\right)^{\frac{p-q}{q}}$, a desigualdade pode ser majorada por:

$\dot{V}(t) \leq 2\left\{\left[S_{G}\left|x_{1}(t)\right|+\left|x_{1}(t)\right|\left|x_{2}(t)\right|+\left|x_{1}(t)\right|\left|x_{3}(t)\right|+S_{G} G_{B}\right.\right.$

$+\frac{1}{t_{\operatorname{maxG}} V}|d(t)|+\frac{1}{\beta} \frac{p}{q}\left(S_{G}\left|x_{1}(t)\right|+\left|x_{1}(t)\right|\left|x_{2}(t)\right|+\left|x_{1}(t)\right|\left|x_{3}(t)\right|\right.$

$\left.+S_{G} G_{B}+\frac{1}{t_{\operatorname{maxG}} V}|d(t)|\right)^{\frac{p-q}{q}}\left(\left(S_{G}^{2}+p_{2} S_{I} I_{B}+p_{3} S_{N} N_{B}\right)\left|x_{1}(t)\right|\right.$

$+S_{G} G_{B}\left(\left|x_{2}(t)\right|+\left|x_{3}(t)\right|\right)+\left(2 S_{G}+p_{2}\right)\left|x_{1}(t)\right|\left|x_{2}(t)\right|$

$+\left(2 S_{G}+p_{3}\right)\left|x_{1}(t)\right|\left|x_{3}(t)\right|+\left|x_{1}(t)\right|\left(\left|x_{2}(t)\right|^{2}+\left|x_{3}(t)\right|^{2}\right)$

$+2\left|x_{1}(t)\right|\left|x_{2}(t)\right|\left|x_{3}(t)\right|+\frac{1}{t_{\operatorname{maxG}} V}\left(S_{G}+\left|x_{2}(t)\right|+\left|x_{3}(t)\right|\right)|d(t)|$

$\left.\left.\left.+\frac{1}{t_{\operatorname{maxG}} V}|\dot{d}(t)|\right)-\frac{1}{\beta} \frac{p}{q}\left(S_{G} G_{B}\right)^{\frac{p-q}{q}} \min \left\{\underline{p}_{2}, \underline{p}_{3}\right\} \min \left\{\underline{S}_{I}, \underline{S}_{N}\right\} \varrho\right]\right\}$.
Utilizando-se os limitantes (17)-(20), tem-se $\dot{V}(t) \leq 2\left\{\left[\bar{S}_{G}\left|x_{1}(t)\right|+\left|x_{1}(t)\right|\left|x_{2}(t)\right|+\left|x_{1}(t)\right|\left|x_{3}(t)\right|+\bar{S}_{G} \bar{G}_{B}\right.\right.$ $+\frac{1}{t_{\operatorname{maxG}} V}|d(t)|+\frac{1}{\beta} \frac{p}{q}\left(\bar{S}_{G}\left|x_{1}(t)\right|+\left|x_{1}(t)\right|\left|x_{2}(t)\right|+\left|x_{1}(t)\right|\left|x_{3}(t)\right|\right.$ $\left.+\bar{S}_{G} \bar{G}_{B}+\frac{1}{t_{\operatorname{maxG}} V}|d(t)|\right)^{\frac{p-q}{q}}\left(\left(\bar{S}_{G}^{2}+\bar{p}_{2} \bar{S}_{I} \bar{I}_{B}+\bar{p}_{3} \bar{S}_{N} \bar{N}_{B}\right)\left|x_{1}(t)\right|\right.$ $+\bar{S}_{G} \bar{G}_{B}\left(\left|x_{2}(t)\right|+\left|x_{3}(t)\right|\right)+\left(2 \bar{S}_{G}+\bar{p}_{2}\right)\left|x_{1}(t)\right|\left|x_{2}(t)\right|$ $+\left(2 \bar{S}_{G}+\bar{p}_{3}\right)\left|x_{1}(t)\right|\left|x_{3}(t)\right|+\left|x_{1}(t)\right|\left(\left|x_{2}(t)\right|^{2}+\left|x_{3}(t)\right|^{2}\right)$ $+2\left|x_{1}(t)\right|\left|x_{2}(t)\right|\left|x_{3}(t)\right|+\frac{1}{t_{\operatorname{maxG}} V}\left(\bar{S}_{G}+\left|x_{2}(t)\right|+\left|x_{3}(t)\right|\right)|d(t)|$ $\left.\left.\left.+\frac{1}{t_{\operatorname{maxG}} V}|\dot{d}(t)|\right)-\frac{1}{\beta} \frac{p}{q}\left(\underline{\mathrm{S}}_{G} \underline{\mathrm{G}}_{B}\right)^{\frac{p-q}{q}} \min \left\{\underline{\mathrm{p}}_{2}, \underline{\mathrm{p}}_{3}\right\} \min \left\{\underline{\mathrm{S}}_{I}, \underline{\mathrm{S}}_{N}\right\} \varrho\right]\right\}$.

Logo,

$$
\begin{aligned}
\dot{V}(t) \leq & 2\left\{\left[\bar{S}_{G}|x(t)|+2|x(t)|^{2}+\bar{S}_{G} \bar{G}_{B}+\frac{1}{t_{\operatorname{maxG}} V}|d(t)|\right.\right. \\
& +\frac{1}{\beta} \frac{p}{q}\left(\bar{S}_{G}|x(t)|+2|x(t)|^{2}+\bar{S}_{G} \bar{G}_{B}+\frac{1}{t_{\operatorname{maxG}} V}|d(t)|\right)^{\frac{p-q}{q}} \times \\
& \left(\left(\bar{S}_{G}^{2}+2 \bar{p}_{2} \bar{S}_{I} \bar{I}_{B}+\bar{p}_{3} \bar{S}_{N} \bar{N}_{B}+2 \bar{S}_{G} \bar{G}_{B}+\frac{1}{t_{\operatorname{maxG}} V}\right)|x(t)|\right. \\
& \left.+\left(4 \bar{S}_{G}+\bar{p}_{2}+\bar{p}_{3}\right)|x(t)|^{2}+4|x(t)|^{3}+\frac{\bar{S}_{G}|d(t)|+|\dot{d}(t)|}{t_{\operatorname{maxG}} V}\right) \\
& \left.\left.-\frac{k_{G}}{\beta} \frac{p}{q}\left(\underline{\mathrm{S}}_{G} \underline{\mathrm{G}}_{B}\right)^{\frac{p-q}{q}} \min \left\{\underline{p}_{2}, \underline{p}_{3}\right\} \min \left\{\underline{S}_{I}, \underline{S}_{N}\right\} \varrho\right]\right\} .
\end{aligned}
$$

Utilizando-se os majorantes (17)-(20), $|x(t)|<\chi,|d(t)|<$ $\bar{d}$ e $|\dot{d}(t)|<\overline{\dot{d}}$ na desigualdade (41), tem-se

$$
\begin{aligned}
\dot{V}(t) \leq & 2\left\{\left[\bar{S}_{G} \chi+2 \chi^{2}+\bar{S}_{G} \bar{G}_{B}+\frac{1}{t_{\operatorname{maxG}} V} \bar{d}\right.\right. \\
& +\frac{1}{\beta} \frac{p}{q}\left(\bar{S}_{G} \chi+2 \chi^{2}+\bar{S}_{G} \bar{G}_{B}+\frac{1}{t_{\operatorname{maxG}} V} \bar{d}\right)^{\frac{p-q}{q}} \times \\
& \left(\left(\bar{S}_{G}^{2}+2 \bar{p}_{2} \bar{S}_{I} \bar{I}_{B}+\bar{p}_{3} \bar{S}_{N} \bar{N}_{B}+2 \bar{S}_{G} \bar{G}_{B}+\frac{1}{t_{\operatorname{maxG}} V}\right) \chi\right. \\
& \left.+\left(4 \bar{S}_{G}+\bar{p}_{2}+\bar{p}_{3}\right) \chi^{2}+4 \chi^{3}+\frac{\bar{S}_{G} \bar{d}+\bar{d}}{t_{\operatorname{maxG}} V}\right) \\
& \left.\left.-\frac{k_{G}}{\beta} \frac{p}{q}\left(\underline{\mathrm{S}}_{G} \underline{\mathrm{G}}_{B}\right)^{\frac{p-q}{q}} \min \left\{\underline{p}_{2}, \underline{p}_{3}\right\} \min \left\{\underline{S}_{I}, \underline{S}_{N}\right\} \varrho\right]\right\} .
\end{aligned}
$$

Definindo-se $\bar{\chi}$ como

$$
\begin{aligned}
& \bar{\chi}=\bar{S}_{G} \chi+2 \chi^{2}+\bar{S}_{G} \bar{G}_{B}+\frac{1}{t_{\operatorname{maxG}} V} \bar{d} \\
& +\frac{1}{\beta} \frac{p}{q}\left(\bar{S}_{G} \chi+2 \chi^{2}+\bar{S}_{G} \bar{G}_{B}+\frac{1}{t_{\operatorname{maxG}} V} \bar{d}\right)^{\frac{p-q}{q}} \times \\
& \times\left(\left(\bar{S}_{G}^{2}+2 \bar{p}_{2} \bar{S}_{I} \bar{I}_{B}+\bar{p}_{3} \bar{S}_{N} \bar{N}_{B}+2 \bar{S}_{G} \bar{G}_{B}+\frac{1}{t_{\operatorname{maxG}} V}\right) \chi\right. \\
& \left.+\left(4 \bar{S}_{G}+\bar{p}_{2}+\bar{p}_{3}\right) \chi^{2}+4 \chi^{3}+\frac{\bar{S}_{G} \bar{d}+\bar{d}}{t_{\operatorname{maxG}} V}\right),
\end{aligned}
$$

tem-se

$$
\dot{V}(t) \leq 2\left\{\left[\bar{\chi}-\frac{k_{G}}{\beta} \frac{p}{q}\left(\underline{\mathrm{S}}_{G} \underline{\mathrm{G}}_{B}\right)^{\frac{p-q}{q}} \min \left\{\underline{p}_{2}, \underline{p}_{3}\right\} \min \left\{\underline{S}_{I}, \underline{S}_{N}\right\} \varrho\right]\right\}
$$

Então, fazendo-se

$$
\varrho=\frac{\bar{\chi}+\delta}{\frac{k_{G}}{\beta} \frac{p}{q}\left(\underline{\mathrm{S}}_{G} \underline{\mathrm{G}}_{B}\right)^{\frac{p-q}{q}} \min \left\{\underline{p}_{2}, \underline{p}_{3}\right\} \min \left\{\underline{S}_{I}, \underline{S}_{N}\right\}},
$$

com $\delta>0$, a derivada de (35) satisfaz a desigualdade

$$
\dot{V}(t) \leq-2 \delta|\sigma(t)|
$$

Definindo-se a variável auxiliar $\tilde{\sigma}(t):=|\sigma(t)|=\sqrt{V(t)}$, tem-se $\dot{\tilde{\sigma}}(t)=\frac{\dot{V}(t)}{2 \sqrt{V(t)}}$. Dividindo ambos os lados de (46) 
por $2 \sqrt{V}(t)$, tem-se

$$
\frac{\dot{V}(t)}{2 \sqrt{V(t)}} \leq-2 \delta \frac{|\sigma(t)|}{2 \sqrt{V(t)}},
$$

que é idêntica a

$$
\dot{\tilde{\sigma}}(t) \leq-\delta, \quad t \geq 0
$$

Usando o teorema da comparação, existe um limitante superior $\bar{\sigma}(t)$ de $\tilde{\sigma}(t)$ satisfazendo a equação diferencial

$$
\dot{\bar{\sigma}}(t)=-\delta, \quad \bar{\sigma}(0)=\tilde{\sigma}(0) \geq 0, \quad t \geq 0 .
$$

Integrando-se os dois lados da equação (49), temos como solução

$$
\bar{\sigma}(t)-\bar{\sigma}(0)=-\delta t \quad t \geq 0 .
$$

Dessa forma, a seguinte igualdade é válida:

$$
\bar{\sigma}(t)=-\delta t+\bar{\sigma}(0), \quad t \geq 0 .
$$

Sabendo que $\bar{\sigma} \geq 0$ é contínuo, então $\sigma(t)$ torna-se identicamente nulo $\forall t \geq t_{1}=\delta^{-1} \bar{\sigma}(0)$. Com isso, podese concluir que existe um tempo finito $0<t_{s} \leq t_{1}$, onde o modo deslizante inicia tal que $\sigma(t)=0$ e $\dot{\sigma}(t)=0$, $\forall t \geq t_{s}$. Além disso, é possível mostrar também que como sigma foi para zero em tempo finito, $e(t)$, e $\dot{e}(t)$ se tornam identicamente nulos em tempo finito, vide Feng et al. (2002).

\section{CONTROLE POR MODOS DESLIZANTES TERMINAL NÃO SINGULAR: VERSÃO CONTÍNUA}

A extensão da realimentação de saída para continuous non-singular terminal sliding mode algorithm (CNTSMA) pode ser obtida seguindo os passos introduzidos por Fridman et al. (2015). Isto pode ser entendido como uma combinação do algoritmo de super-twisting Gonzalez et al. (2012) com SMC terminal não-singular acima e pode ser escrita como:

$$
\begin{aligned}
u= & -\left.\left.k_{1}\left|\zeta_{1}+k_{2}\right| e\right|^{2 / 3} \operatorname{sgn}(e)\right|^{1 / 2} \operatorname{sgn}\left(\zeta_{1}+k_{2}|e|^{2 / 3} \operatorname{sgn}(e)\right) \\
& -k_{3} \int_{0}^{t} \operatorname{sgn}\left(\zeta_{1}+k_{2}|e|^{2 / 3} \operatorname{sgn}(e)\right) d \tau
\end{aligned}
$$

onde $k_{1}>0, k_{2}>0$ e $k_{3}>0$ são constantes apropriadas.

\section{RESULTADOS DE SIMULAÇÃO}

Nesta seção, apresentam-se os resultados de simulação referentes aos controladores discutidos nas seções 6 e 7 .

\subsection{Controlador por Modos Deslizantes Terminal Não-Singular: Versao Descontínua}

Observe a Figura 3 a seguir.

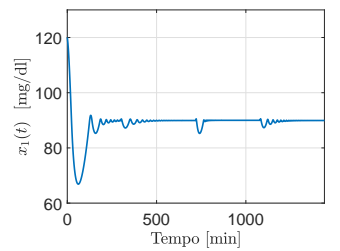

(a) Curva glicêmica

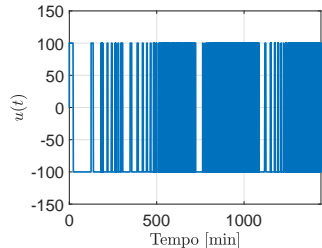

(b) Ação do controlador.

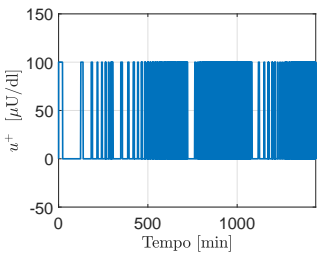

(c) Curva da Insulina.

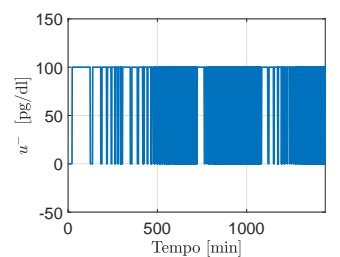

(d) Curva do Glucagon.

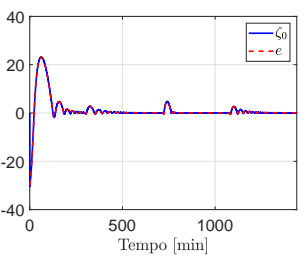

(e) Comparando $\zeta_{0} \operatorname{com} e$

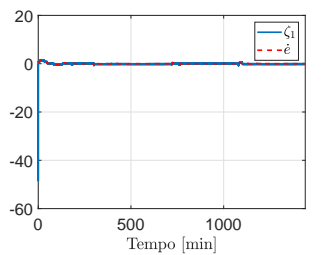

(f) Comparando $\zeta_{1} \operatorname{com} \dot{e}$.
Figura 3. - Curvas referentes à versão descontínua do controlador por modos deslizantes terminal não-singular.

A partir da Figura 3(a), observa-se que o desempenho do controlador é aceitável. Nenhum caso de hipoglicemia ou de hiperglicemia é evidenciado ao longo do período avaliado. A Figura 3(b) ilustra a ação descontínua do controlador e a presença de chattering. Deve-se destacar a amplitude elevada da ação de controle. Esta amplitude se deve ao fato dos ganhos do controlador em (33) serem bastante altos. A consequência disto é a utilização de uma quantidade muito grande de hormônios sendo injetada no paciente a todo tempo. Este efeito, obviamente, não é desejável, uma vez que pode vir a causar danos à saúde do paciente.

As Figuras 3(c) e 3(d) demonstram o papel do atuador bi-hormonal, separando a ação de controle positiva, $u^{+}$, e a ação de controle negativa, $u^{-}$, em duas ações isoladas e independentes. A ação de controle positiva representa a injeção de insulina no organismo do paciente com DMT1, enquanto a ação de controle negativa representa a injeção de glucagon.

As Figuras 3(e) e 3(f) atestam a favor da brevidade do transitório das variáveis $\zeta_{0}$ e $\zeta_{1}$. Em outras palavras, dentro de pouco tempo, as estimativas de $e$ e de $\dot{e}$ são perfeitamente obtidas. Desse modo, a variável ideal de deslizamento $\sigma$ pode ser construída a partir de $\hat{\sigma}$, garantindo que o deslizamento ideal $\sigma=0$ seja alcançado. Cabe dizer que as grandezas ilustradas na Figura 3(e) são medidas em $[\mathrm{mg} / \mathrm{dl}]$, enquanto que as grandezas representadas em Figura 3(f) são medidas em $\left[\mathrm{mg} \mathrm{dl}^{-1} \mathrm{~min}^{-1}\right]$.

Os resultados de simulação referentes à versão contínua do controlador por modos deslizantes terminal não-singular apresentarão uma série de semelhanças com os resultados discutidos nesta seção. Por este motivo, a fim de se evitar a repetição de conteúdo, a seção a seguir apresentará comentários mais breves, feitos sempre em referência aos comentários supracitados. 

8.2 Controle por Modos Deslizantes Terminal Não Singular:
Versão Contínua

Os resultados de simulação referentes ao controlador CNTSMA são apresentados a seguir.

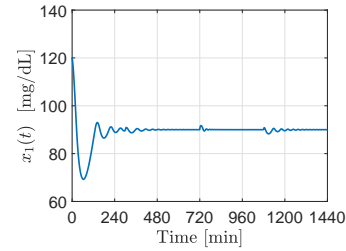

(a) Curva glicêmica.

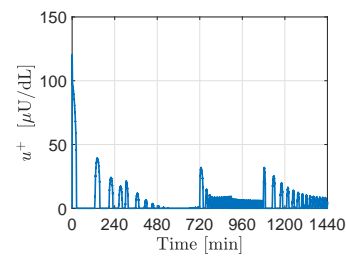

(c) Curva da Insulina.

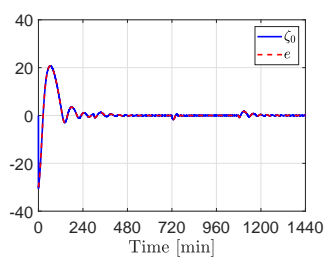

(e) Comparando $\zeta_{0}$ com $e$.

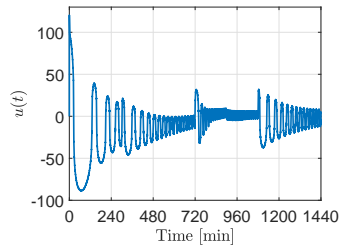

(b) Ação do controlador.

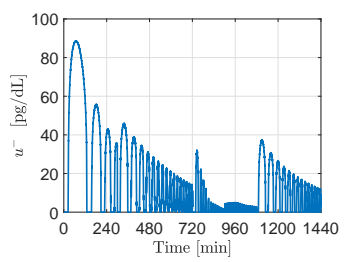

(d) Curva do Glucagon.

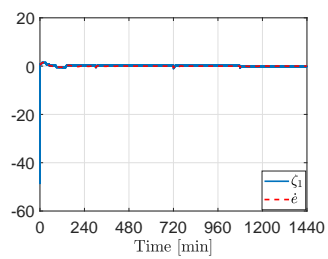

(f) Comparando $\zeta_{1}$ com $\dot{e}$.
Figura 4. - Curvas referentes à versão contínua do controlador por modos deslizantes terminal não-singular.

A partir da Figura 4(a), observa-se que, uma vez mais, o desempenho do controlador é aceitável. Além disso, observa-se na Figura 4(b) que o efeito chattering foi eliminado da ação de controle $u(t)$. Em outras palavras, a ação de controle passou a ser descrita por uma função suave, diferente da ação de controle descontínua evidenciada na seção 6. Isto se deve à utilização da versão contínua do controlador por modos deslizantes terminal não-singular. Nos demais aspectos, destaca-se o mesmo que foi comentado na seção 6.

\section{CONCLUSÃO}

Neste projeto, um controlador por modos deslizantes terminal não singular foi utilizado com o intuito de garantir a regulação glicêmica em um paciente diabético tipo 1. Do ponto de vista de controle, este é um problema desafiador, tendo em vista que o processo é representado por uma planta não-linear, variante no tempo, onde os parâmetros foram considerados incertos. Trata-se de um caso onde se faz presente uma perturbação descasada, representada por $d(t)$. Além disso, a saída do sistema possui grau relativo $\rho=2$ no que diz respeito à ação controle, e por se tratar de um sistema biológico, o problema de controle deve ser tratado como uma estratégia estritamente positiva.

Estes problemas foram contornados utilizando-se majorantes paramétricos e limitantes superiores para as perturbações, um atuador bi-hormonal e a teoria de controle por modos deslizantes. Os resultados de simulação demonstram que as estratégias de controle utilizadas se mostraram eficientes no processo de regulação glicêmica.

\section{REFERÊNCIAS}

Bakhtiani, P., Zhao, L., Youssef, J.E., Castle, J., and Ward, W. (2013). A review of artificial pancreas technologies with an emphasis on bi-hormonal therapy. In Diabetes, Obesity and Metabolism, 1065-1070. John Wiley and Sons.

Bequette, B.W. (2012). Challenges and recent progress in the development of a closed-loop artificial pancreas. Annual Reviews in Control, 36, 255-266.

Castle, J., Engle, J., Youssef, J.E., Massoud, R., Yuen, K., Kagan, R., and Ward, W. (2010). Novel use of glucagon in a closed-loop system for prevention of hypoglycemia in type 1 diabetes. Diabetes Care, 33, 1282-1287.

Cobelli, C., Renard, E., and Kovatchev, B. (2011). Artificial pancreas: Past, present, future. Diabetes, 60, 26722682.

Edwards, C. and Spurgeon, S. (1998). Sliding Mode Control: Theory and Applications. CRC Press.

El-Khatib, F., Russell, S., Nathan, D., Sutherlin, R., and Damiano, E. (2010). A bihormonal closed-loop artificial pancreas for type 1 diabetes. Science Translational Medicine, 2, $27 \mathrm{ra} 27$.

Feng, Y., Yu, X., and Man, Z. (2002). Non-singular terminal sliding mode control of rigid manipulators. Automatica, 38, 2159-2167.

Fridman, L., Moreno, J., and Bandyopadhyay, B. (2015). Recent Advances in Sliding Modes: From Control to Intelligent Mechatronics, Editors: X. Yu and M. O. Efe. Springer-Verlag.

Gonzalez, T., Moreno, J.A., and Fridman, L. (2012). Variable gain super-twisting sliding mode control. IEEE Transactions on Automatic Control, 57(8), 2100-2105.

Guyton, A. and Hall, J. (2016). Textbook of Medical Physiology. Elsevier.

Herrero, P., Georgiou, P., Oliver, N., Reddy, M., Johnston, D., and Toumazou, C. (2013). A composite model of glucagon-glucose dynamics for in silico testing of bihormonal glucose controllers. Journal of Diabetes Science and Technology, 7, 941-951.

Kadish, A. (1964). Automation control of blood sugar. servomechanism for glucose monitoring and control. The American journal of medical electronics, 9, 82-86.

Kaveh, P. and Shtessel, Y. (2008). Blood glucose regulation using higher-order sliding mode control. International Journal of Robust and Nonlinear Control, 18, $557-569$.

Levant, A. (2003). Higher-order sliding modes, differentiation and output-feedback control. International Journal of Control, 76, 924-941.

Russell, S., El-Khatib, F., Nathan, D., Magyar, K., Jiang, J., and Damianod, E. (2012). Blood glucose control in type 1 diabetes with a bihormonal bionic endocrine pancreas. Diabetes Care, 35, 2148-2155. 